\title{
A Long-Term View at Clipping Versus Coiling for Ruptured Aneurysm
}

\author{
Alejandro A. Rabinstein
}

Published online: 9 June 2009

(C) Humana Press Inc. 2009

The results of the long-term follow-up of the ISAT patients are mostly confirmatory [1]. ISAT remains the most influential trial comparing clipping versus coiling of ruptured aneurysms. Only cases that fulfilled the condition of clinical equipoise were enrolled. In other words, only patients who were considered adequate candidates for clipping and for coiling were randomized. The initial report of the results of the study indicated that coiling was associated with a relative reduction in the risk of death and dependency at 1 year of $23.9 \%$ compared with clipping (absolute risk reduction 7.4\%) [2]. During the first year after treatment, coiled patients had a slightly higher incidence of rebleeding from the treated aneurysm and required re-treatment (typically additional coiling) 6.9 times more often than clipped patients [2,3]. However, there were concerns about the durability of coiling in patients who are often quite young at the time of their subarachnoid hemorrhage.

During a mean follow-up of 9 years (range 6-14 years), the risk of rebleeding from the treated aneurysm remained low in both groups, although it was higher in the coiling group (the difference was actually significant in the per protocol analysis, which accounted for a patient assigned to the clipping group who requested to be coiled instead and later rebled). It is possible that current risk of aneurysm rebleeding after coiling could be lower than those seen in ISAT because technological improvement now allows higher rates of complete aneurysm obliteration, but this possibility remains to be proven. There were no differences in fatal cases of rebleeding between the two groups.

A. A. Rabinstein $(\bowtie)$

Mayo Clinic, 200 First Street SW, Rochester, MN 55905, USA

e-mail: rabinstein.alejandro@mayo.edu
Clipping did not eliminate the risk of late rebleeding from the treated aneurysm. Bleeding could also occur from another previously known but untreated aneurysm or from aneurysms which formed de novo after enrollment in the trial. Table 1 shows the distribution of cases of rebleeding according to bleeding source and treatment arm.

The risk of death at 5 years was significantly lower in the coiled group (relative risk 0.77). Yet, the chances of independent survival for those patients surviving 5 years were similar in both groups. This apparent discrepancy is probably explained by functional improvement of patients with initial moderate disability in both groups and the demise of some patients with severe disability which had contributed to the difference in functional outcome among survivors at 1 year. Notably, the mortality rate of trial patients surviving more than 1 year was higher than the mortality expected for the general population (standardized mortality rate 1.57 ).

There are several important lessons to be learned from this follow-up data. All patients with subarachnoid hemorrhage should have follow-up angiograms, especially if they are smokers or have hypertension (unfortunately these two risk factors for aneurysm rupture and de novo aneurysm formation were not collected as part of ISAT). The timing of repeat angiograms, duration of angiographic monitoring, and relative value of non-invasive versus catheter angiograms in clipped and coiled patients need to be defined by future studies. The cost-effectiveness of angiographic monitoring should also be analyzed. As correctly pointed out by the study investigators and the accompanying editorial [3], coiling has benefits over clipping in patients who are adequate candidates for both treatments, but multiple factors (e.g., anatomical features of the aneurysm, local expertise in each of the treatment options, age, and comorbid conditions of the patient), need 
Table 1 Recurrent cases of subarachnoid hemorrhage occurring 1 year or more after treatment allocation in ISAT

\begin{tabular}{llllrr}
\hline & $\begin{array}{l}\text { Rebleeding from } \\
\text { treated aneurysm }\end{array}$ & $\begin{array}{l}\text { Rebleeding from other } \\
\text { aneurysm seen at baseline }\end{array}$ & $\begin{array}{l}\text { Bleeding from de } \\
\text { novo aneurysm }\end{array}$ & $\begin{array}{l}\text { Unknown source } \\
\text { of bleeding }\end{array}$ \\
\hline Coiling & 10 & 3 & 3 & 1 & 17 \\
Clipping & $3^{\text {a }}$ & 1 & 3 & 0 & 7 \\
Total & 13 & 4 & 6 & 1 & 24 \\
\hline
\end{tabular}

a 1 patient was actually a cross-over (i.e., had been coiled)

to be weighed in the decision of which treatment modality should be chosen to secure the aneurysm.

\section{References}

1. Molyneux AJ, Kerr RS, Birks J, Ramzi N, Yarnold J, Sneade M, et al. Risk of recurrent subarachnoid haemorrhage, death, or dependence and standardised mortality ratios after clipping or coiling of an intracranial aneurysm in the International Subarachnoid Aneurysm Trial (ISAT): long-term follow-up. Lancet Neurol. 2009;8:427-33.
2. Molyneux AJ, Kerr RS, Yu LM, Clarke M, Sneade M, Yarnold JA, et al. International subarachnoid aneurysm trial (ISAT) of neurosurgical clipping versus endovascular coiling in 2143 patients with ruptured intracranial aneurysms: a randomised comparison of effects on survival, dependency, seizures, rebleeding, subgroups, and aneurysm occlusion. Lancet. 2005;366:809-17.

3. Campi A, Ramzi N, Molyneux AJ, Summers PE, Kerr RS, Sneade $\mathrm{M}$, et al. Retreatment of ruptured cerebral aneurysms in patients randomized by coiling or clipping in the International Subarachnoid Aneurysm Trial (ISAT). Stroke. 2007;38:1538-44.

4. Broderick J. Clipping or coiling: the first step for ruptured aneurysms. Lancet Neurol. 2009;8:414-5. 